DOCTRINA

\title{
La esencialidad del principio de responsabilidades comunes pero diferenciadas en el régimen internacional del cambio climático
}

\author{
The essentiality of the principle of common but differentiated \\ responsibilities in the international climate change regime
}

\author{
Pedro Cisterna Gaete (iD \\ Universidad de Edimburgo, Escocia
}

\begin{abstract}
RESUMEN Este artículo revisa la esencialidad del principio de responsabilidades comunes pero diferenciadas en el desarrollo del régimen internacional del cambio climático. El análisis repasa la evolución que el principio ha tenido desde su incorporación en la Convención Marco de las Naciones Unidas para el Cambio Climático y la relevancia de su rol en el progreso actual del régimen climático. Primero, se desarrolla un análisis general del principio, con foco en su origen. Segundo, se revisa la legitimidad del principio de responsabilidades comunes pero diferenciadas dentro del régimen climático. A continuación, el análisis brinda ciertas respuestas a las críticas que ha recibido este principio. Finalmente, se elaboran comentarios y proyecciones sobre el estado actual y futura relevancia del principio de responsabilidades comunes pero diferenciadas en el progreso del régimen climático.
\end{abstract}

PALABRAS CLAVE Derecho internacional del cambio climático, principio de responsabilidades comunes pero diferenciadas, equidad, Acuerdo de París, derecho ambiental internacional.

ABSTRACT The work aims to analyse the essentiality of the common but differentiated principle within the international climate change regime. This examination is developed by studying the evolution of the principle from its incorporation to the United Framework Convention on Climate Change to its current relevance in the progress of the climate change regime. The article firstly develops a general analysis of the principle, mainly focused on its origin. Then, the paper examines the legitimacy of the common but differentiated responsibilities principle within the climate change regime. Subsequently, the analysis presents some answers to the critics received by this principle. Finally, the article delivers some comments and projections regarding the current and 
future relevance of the principle of common but differentiated responsibilities in the progress of the climate change regime.

KEYWORDS International climate change law, principle of common but differentiated responsibilities, equity, Paris Agreement, international environmental law.

\section{Introducción}

El principio de responsabilidades comunes pero diferenciadas es un elemento esencial en la evolución y desarrollo del régimen internacional del cambio climático (Bodansky, Brunnée y Rajamani, 2017: 26). Esto se debe principalmente a que incorpora un criterio de equidad en las acciones para enfrentar el fenómeno (Rajamani, 2012: 152). Sin embargo, la interpretación e implementación de este principio no ha estado exenta de debates y críticas (French, 2000: 36).

En este contexto, este artículo busca demostrar la esencialidad del principio de responsabilidades comunes pero diferenciadas en el funcionamiento y desarrollo del régimen internacional del cambio climático, analizando su evolución dentro de este régimen y destacando algunos de sus aspectos relevantes de cara a la próxima Conferencia de las Partes (COP) 26. En esta línea, primero, este ensayo desarrollará un enfoque general del principio de responsabilidades comunes pero diferenciadas. Posteriormente, analizará la importancia de este principio desde los orígenes del régimen internacional del cambio climático, planteando argumentos éticos y prácticos. Asimismo, se abordarán las críticas que este principio ha recibido durante su implementación y como las ha sobrellevado. En este sentido, este artículo se enfocará en el camino que ha recorrido la interpretación de este principio hacia el logro del Acuerdo de París.

Considerando los presupuestos anteriores, el texto examinará las reuniones climáticas posteriores al Acuerdo de París (ONU, 2016) y su tratamiento del principio de responsabilidades comunes pero diferenciadas, destacando su rol en la próxima $\mathrm{COP} 26$. Este rol se ilustrará analizando el papel de la ciencia en el régimen internacional del cambio climático y la implementación del artículo 6 del Acuerdo de París.

\section{Perspectivas generales del principio de responsabilidades comunes pero diferenciadas}

Esta sección identificará los principales elementos del principio, buscando establecer una base común sobre la que este trabajo desarrollará su análisis.

La equidad es un principio pilar y transversal del régimen internacional del cambio climático y está consagrado en el artículo 3.1 de la Convención Marco de las Naciones Unidas para el Cambio Climático (CMNUCC), adoptada en mayo de 1992 y 
que entró en vigencia en marzo de 1994. El principio de equidad contiene una distinción: equidad intergeneracional y equidad intrageneracional. La primera se refiere a la protección de las futuras generaciones, y establece el deber de las generaciones presentes de no dejar el planeta en un estado peor del que lo recibieron. ${ }^{1}$ Por otra parte, equidad intrageneracional se refiere a aquella enfocada en las generaciones presentes ${ }^{2}$ y se vincula con el desarrollo sustentable y la erradicación de la pobreza. ${ }^{3}$

Se afirma que a nivel interestatal el principio de responsabilidades comunes pero diferenciadas es una expresión de equidad intrageneracional. En particular, el principio propone una perspectiva diferenciada (AGNU, 1989a, 1989b) entre los Estados miembros de la Convención. Esto constituye una vía original para determinar obligaciones y compromisos sustentados en una preocupación común, ${ }^{4}$ asumiendo las distintas necesidades y prioridades de países desarrollados y países en vías de desarrollo (Brunnée y Streck, 2013).

Este principio está reconocido en distintos instrumentos de derecho internacional (Rajamani, 2012: 129). Por ejemplo, la Declaración de Estocolmo (ONU, 1972) estableció la necesidad de transferir recursos y tecnología desde países desarrollados hacía países en vías de desarrollo con el objeto de cumplir con estándares ambientales. ${ }^{5}$ Además, es mencionado en los principios sexto y séptimo de la Declaración de Río (AGNU, 1992). De hecho, el principio séptimo de dicha Declaración reconoce expresamente el principio de responsabilidades comunes pero diferenciadas, y señala dos aspectos de diferenciación: «la contribución de la degradación ambiental y la capacidad/recursos para tomar medidas de respuesta» (Rajamani, 2012: 130).

En sus orígenes, el principio de responsabilidades comunes pero diferenciadas nació desde la noción de - valga la redundancia - responsabilidad común para la protección del medio ambiente (Sands, 2003), que descansa en la idea de la preocupación común (Soltau, 2016). Esta visión implica compromisos colectivos e individuales diseñados bajo una perspectiva de cooperación, donde la responsabilidad común puede ser entendida como el paraguas a través del cual las responsabilidades colectivas e individuales son definidas.

A la luz de la noción de preocupación común, el criterio diferenciador de las responsabilidades climáticas debiese considerar dos aspectos. Por una parte, la responsabilidad histórica (Rajamani, 2012: 138) de los países desarrollados, quienes están llamados a considerar las externalidades negativas (Shue, 1999) provocadas por ellos mismos (Mickelson, 2008). Por otra parte, las capacidades (Bodansky, Brunnée y Ra-

\footnotetext{
1. Véase Brown (2007), Weston (2007). También CMNUCC, preámbulo párrafo final.

2. Véase CMNUCC, artículo 3.1 y artículo 3.2.

3. Véase CMNUCC, artículo 3.5.

4. Véase CMNUCC, preámbulo párrafo inicial.

5. En este sentido, veéase la Declaración de Estocolmo (1972), principios 9, 12 y 20.
} 
jamani, 2017: 27) de cada nación para tomar medidas de mitigación y adaptación que enfrenten el cambio climático. Esto último implica asumir una perspectiva más dinámica de los esfuerzos que cada país podría impulsar, dado que las capacidades de cada país pueden variar en el tiempo.

Además de las perspectivas anteriores, existen posturas que sostienen un criterio más matizado de diferenciación (Atapattu, 2009: 37). ${ }^{6}$ Algunos afirman que cuando países en vías de desarrollo alcanzan cierta estabilidad económica o han alcanzado un significativo número de emisiones, la definición de sus obligaciones debería apartar cualquier tipo de diferenciación (Atapattu, 2009: 44). Otros argumentan que el principio de responsabilidades comunes pero diferenciadas se ha extendido a cuestiones ambientales de derecho interno, principalmente en favor de los pueblos indígenas y de poblaciones vulnerables (Atapattu, 2009: 58).

Considerando lo anterior, dentro de este principio es posible identificar ciertos elementos: responsabilidad común, cooperación, contribución histórica y capacidades (Rajamani, 2012: 133). Estos son los factores con que la diferenciación es aplicada bajo los enfoques antes analizados. En general, su incorporación supone un avance sustantivo en el reconocimiento de las realidades nacionales para enfrentar desafíos globales. Ciertamente, la implementación y recorrido de este principio en el régimen climático puede contribuir al diseño de modelos de cooperación similares en materias nacionales o internacionales que supongan esfuerzos colectivos de múltiples partes.

\section{La legitimidad del principio de responsabilidades comunes pero diferenciadas desde un aspecto histórico, ético y práctico}

Esta sección busca justificar la legitimidad del principio de responsabilidades comunes pero diferenciadas dentro del régimen internacional del cambio climático. Para ello, se analizarán las negociaciones con que el principio fue incorporado a la Convención Marco de las Naciones Unidas para el Cambio Climático. Además, esta sección argumentará razones éticas y prácticas para sostener la esencialidad del principio de responsabilidades comunes pero diferenciadas dentro del régimen internacional del cambio climático.

El inicio de la incorporación del principio de responsabilidades comunes pero diferenciadas dentro del régimen internacional del cambio climático

El principio de responsabilidades comunes pero diferenciadas es parte del régimen internacional del cambio climático. La Convención Marco establece el tratamiento

6. Véase el artículo 7 del Acuerdo de París sobre adaptación y la especial consideración sobre poblaciónes vulnerables. 
diferenciado en su preámbulo, considerando las emisiones históricas de los países desarrollados y las necesidades especiales de los países en desarrollo. ${ }^{7}$ El artículo 3.1 de la Convención reconoce oficialmente el principio de responsabilidades comunes pero diferenciadas, ${ }^{8}$ afirmando que los países desarrollados deben tomar el liderazgo para afrontar el cambio climático.

Las intenciones de incorporar un tratamiento diferenciado (Rajamani, 2012: 129) en la Convención Marco de las Naciones Unidas para el Cambio Climático aparecieron durante sus negociaciones preliminares. En la primera sesión del Comité Intergubernamental de Negociación (AGNU, 1991a), varias declaraciones insistieron en la importancia de transferir recursos desde países desarrollados hacia países en desarrollo con el objetivo de que estos últimos pudieran financiar políticas públicas en materia de cambio climático. Estas declaraciones, durante la tercera sesión del Comité Intergubernamental de Negociación, se tornaron en un «enorme apoyo» (AGNU, 1991b: par. 76) a la inclusión del principio en el texto definitivo de la Convención. Este acuerdo por la inclusión del principio se sostuvo a pesar de las divergencias respecto de la sección en la cual los «principios» debían ser incorporados. Además, la tercera sesión también hizo relevantes aportes acerca de la flexibilidad del principio de responsabilidades comunes pero diferenciadas. Evitando establecer una distinción severa entre los países desarrollados y los países en vías de desarrollo, la Comisión se enfocó en la situación de las economías en transición y los pequeños estados isleños (AGNU, 1991b: pár. 85). Posteriormente, la cuarta sesión del Comité de Negociación Intergubernamental avanzó en la incorporación del principio en la Convención a través del Documento de Trabajo Consolidado (AGNU, 1991c). Aun cuando la diferenciación era mencionada en el preámbulo de este documento, había incerteza respecto de su naturaleza, es decir, si esta diferenciación estaba basada en responsabilidades o capacidades. Así, el Documento de Trabajo Consolidado reconoció el principio de responsabilidades comunes pero diferenciadas bajo sus dos nociones, responsabilidad y capacidad, y no optó por ninguna en particular. Dicha postura fue finalmente confirmada en el texto final del artículo 3.1 de la Convención Marco, que terminó reconociendo ambas perspectivas.

El análisis de las sesiones del Comité Intergubernamental de Negociación refleja la relación intrínseca que ha existido entre el principio de responsabilidades comunes pero diferenciadas y el régimen internacional del cambio climático desde los orígenes de este último. Adicionalmente, este análisis ilustra que este principio ha sido siempre discutido e interpretado con variaciones, lo cual es normal en cualquier tipo

7. CMNUCC, Preámbulo.

8. Es relevante que el principio es también reconocido en varios pasajes de la CMNUCC, específicamente el artículo 4, relacionado con los «compromisos». Véase en este sentido Brunnée y Streck, 2013: 592-593. 
de negociación ambiental (Carroll, 1988: 13). También, esto nos ayuda a entender los distintos modos de implementación que ha tenido a través del desarrollo del régimen climático. El análisis de estas negociaciones preliminares refleja el rol bisagra de este principio en otros acuerdos logrados por el Comité, lo que ilustra la esencialidad que el principio ha tenido desde el comienzo de las negociaciones de la Convención.

\section{La legitimación ética de la incorporación del principio en el régimen internación del cambio climático}

Como ya fue establecido, el cambio climático es una preocupación común de la humanidad (Soltau, 2016), dado que afecta distintos aspectos de nuestras vidas y nuestro planeta (Stern, 2010: 40; IPCC, 2018: 9-13). En este sentido, algunos intelectuales han sostenido que el cambio climático implica un «cambio paradigmático de la ética». ${ }^{9}$ Por tanto, existiría un deber moral de prevenir o disminuir sus efectos (Gardiner, 2004: 14).

En este orden, un importante debate en ética está relacionado con la responsabilidad y proporción con que estas acciones deben ser distribuidas entre los múltiples actores involucrados. Bajo el contexto del «cambio climático como un problema ético» (Bodansky, Brunnée y Rajamani, 2017: 7), algunos demandan que los países desarrollados lideren las acciones en esta materia (Gardiner, 2004: 14). Es en este punto donde el principio de responsabilidades comunes pero diferenciadas juega un aspecto esencial: establece las directrices bajo las cuales dichas cargas son distribuidas, y empuja a que sean los países desarrollados los que asuman el costo de las acciones climáticas en favor de los países menos desarrollados.

Sin embargo, existe falta de acuerdo en la justificación de este tratamiento diferenciado entre países desarrollados y en vías de desarrollo. Mientras los países en vías de desarrollo se han identificado con el argumento de las responsabilidades derivadas de las emisiones históricas, los países desarrollados han optado por sustentar una diferenciación basada en capacidades (Bodansky, Brunnée y Rajamani, 2017: 27; Brunnée y Streck, 2013: 590).

Considerando estas visiones, proponemos lo siguiente respecto del argumento que sustenta el tratamiento diferenciado (Rajamani, 2012: 129). Reducir el argumento de la diferenciación a una cuestión de capacidades (Bodansky, Brunnée y Rajamani, 2017: 27). implicaría asumir el principio de responsabilidades comunes pero diferenciadas como un gesto de solidaridad, un gesto de caridad desde los países desarrollados hacia los países en vías de desarrollo. Esto supone ignorar las consecuencias de acciones previas de naciones desarrolladas, debilitando el deber moral de tener un comportamiento vigilante y cuidadoso respecto del cambio climático (Shue, 2016: 182).

9. Jameson Dale (2003) citado en Gardiner (2004). Véase también Papa Francisco, Laudati Si' sobre el cuidado de la casa común (2005), disponible en https://bit.ly/3fYQMQ7. 
En el sentido contrario, si se restringe la diferenciación tan solo a una perspectiva histórica, se obstruye la consideración de las transformaciones económicas, ambientales, sociales o políticas que las naciones puedan sufrir en el futuro. Por tanto, la justificación de la diferenciación debe ser entendida desde una perspectiva dual, que incluya ambas nociones, responsabilidades y capacidades. Este fundamento incluiría dos elementos: primero, que las naciones desarrolladas deben asumir su responsabilidad por las emisiones históricas que han causado esta emergencia climática; y segundo, que los países desarrollados, considerando sus capacidades, se comprometan a cooperar con las naciones en vías de desarrollo para estas puedan cumplir con sus respectivos desafíos en materia climática.

Entendiendo el principio de responsabilidades comunes pero diferenciadas desde esta perspectiva dual, se refuerza el régimen internacional del cambio climático porque se establece una lógica de responsabilidades y cooperación en vez de una pura asistencia o mera culpabilidad. Además, la implementación de esta perspectiva permitirá al régimen de cambio climático también demandar cooperación a los países en vías de desarrollo que eventualmente se conviertan en países desarrollados, como es el caso de China.

La perspectiva anterior permite afirmar que la incorporación del principio en el régimen internacional del cambio climático es esencial en el desarrollo de un sistema de responsabilidades continuas, que considera las contribuciones históricas al calentamiento global y las presentes y futuras capacidades de cada nación. Así, este sistema de responsabilidades continuas, sustentado por el principio de responsabilidades comunes pero diferenciadas, se constituye como un motor de compromisos nacionales para el establecimiento e implementación de acciones de mitigación y adaptación.

\section{La legitimidad de la incorporación del principio desde una perspectiva práctica}

La legitimidad práctica de la incorporación del principio de responsabilidades comunes pero diferenciadas dentro del régimen internacional del cambio climático se justifica en dos argumentos: en su naturaleza como principio del derecho ambiental internacional y en la relevancia táctica que tiene en el logro de los objetivos establecidos en la Convención Marco.

Respecto del primer argumento, los principios del derecho internacional son relevantes en la «interpretación, aplicación y desarrollo de los tratados» (Birnie, Boyle y Redgwell, 2009: 28). Adicionalmente, estos principios constituyen un «instrumento de acción flexible» (Dworkin citado por De Sadeleer, 2002: 307) dada su adaptabilidad a diferentes circunstancias y contextos (De Sadeleer, 2002: 307). Como principio, las responsabilidades comunes pero diferenciadas contienen estos elementos, $y$ constituyen una herramienta legal que orienta el logro de los objetivos del régimen internacional del cambio climático (Brunnée y Streck, 2013: 602) y guían la tarea de 
distribuir las acciones climáticas que cada nación debe asumir (Bodansky, Brunnée y Rajamani, 2017: 52). Ciertamente, la esencialidad de estas funciones para el desarrollo del régimen internacional del cambio climático legitima la inserción del principio responsabilidades comunes pero diferenciadas en él.

Por otra parte, el principio siempre ha tenido un rol estratégico en las negociaciones de la Convención Marco para el Cambio Climático (van der Gaast, 2017: 53). Uno de los campos de negociación más difíciles en el Comité Intergubernamental de Negociaciones de la Convención fue la determinación de las responsabilidades históricas respecto del cambio climático (van der Gaast, 2017: 44). En este sentido, la adopción del principio fue una «táctica decisiva» para lograr el acuerdo global que supuso la propia Convención (van der Gaast, 2017: 53). El valor determinante del principio tiene relación con las necesarias negociaciones para el logro de un equilibrio entre la constitución de instrumentos internacionales efectivos de protección ambiental y un amplio apoyo de la comunidad internacional (van der Gaast, 2017: 49).

Los dos puntos anteriores nos permiten demostrar que el principio de responsabilidades comunes pero diferenciadas es un acuerdo esencial, y sin el cual la adopción de la Convención Marco de las Naciones Unidas para el Cambio Climático pudo haberse visto amenazada en su efectividad o incluso en su existencia.

\section{Abordando las críticas: ¿Es el principio de responsabilidades comunes pero diferenciadas una desventaja para el régimen internacional del cambio climático?}

Esta sección abordará alguna de las críticas que ha recibido la adopción y aplicación del principio de responsabilidades comunes pero diferenciadas en el régimen internacional del cambio climático. Estas críticas derivan de la aplicación que el Protocolo de Kioto le dio a este principio, que diferenció estrictamente entre obligaciones impuestas a países desarrollados y países en vías de desarrollo (Bodansky, Brunnée y Rajamani, 2017: 166). En esta sección argumentamos que el Protocolo de Kioto representa solo una interpretación del principio y no su naturaleza misma. Así, sostenemos que la naturaleza de este principio es flexible y adaptable, y permite su permanente mejora.

El Mecanismo de Desarrollo Limpio y el Protocolo de Kioto: Una herramienta flexible en un protocolo inflexible

El Protocolo de Kioto para la Convención Marco de las Naciones Unidas para el Cambio Climático, adoptado en diciembre de 1997 y que entró en vigor en 2005, fue la primera implementación del principio de responsabilidades comunes pero dife- 
renciadas en el régimen internacional del cambio climático. En dicho instrumento se sostuvo una interpretación muy literal de este principio (Weisslitz, 2002). Se estableció una fuerte diferenciación entre los países del Anexo I y los países que no se encuentran en dicho anexo: se establecieron compromisos de reducción de emisiones sólo para las naciones listadas en ese documento (Bodansky, Brunnée y Rajamani, 2017: 166).

Algunas visiones críticas aparecieron respecto de esta estricta diferenciación, y que argumentaron que era un error priorizar las preocupaciones económicas de los países en vías de desarrollo sobre el cuidado ambiental en un contexto donde ambos son igualmente relevantes (Weisslitz, 2002: 489). Estas posturas resaltaron el efecto económico regresivo que esto traería a países en vías de desarrollo, dado el alto costo de adaptación hacia economías sustentables que estas naciones pagarían luego de desarrollar un crecimiento basado principalmente en combustibles fósiles (Stewart, 1992, citado Weisslitz, 2002: 489).

No obstante las perspectivas anteriores, es necesario recordar que el principio se erige bajo una noción de cooperación (Rajamani, 2012: 120-121; Shelton, 2008: 662), por tanto su aplicación debe considerar la preocupación común que el cambio climático implica (Soltau, 2016). En esta línea, el Protocolo de Kioto incluye el Mecanismo de Desarrollo Limpio en su artículo 12, instrumento de cooperación elaborado por la delegación brasileña en los últimos días de negociaciones del protocolo (Yamin, 1998). A través de este mecanismo, naciones desarrolladas podían generar proyectos sustentables en países en vías de desarrollo y obtener certificados de reducción de emisiones. Además, este mecanismo habilitaría a las naciones no desarrolladas, considerando sus limitaciones económicas, a construir caminos más sustentables de crecimiento (Werksman, 1998).

El Mecanismo de Desarrollo Limpio es una expresión del principio de responsabilidades comunes pero diferenciadas. Este instrumento superó la restrictiva diferenciación del protocolo a través de la flexibilidad y adaptabilidad que el principio otorga. Los elementos de este principio contribuyeron a que, aun bajo un instrumento restrictivo como el Protocolo de Kioto, se alcanzaran los objetivos del régimen internacional del cambio climático bajo una noción de responsabilidad común (Rajamani, 2012: 133). Así, el Mecanismo de Desarrollo Limpio, que fue diseñado bajo la noción de responsabilidades comunes pero diferenciadas, representa un instrumento flexible dentro de un protocolo inflexible. El Mecanismo ilustra la adaptabilidad del principio, y contribuye a la creación de soluciones innovadoras y que pluralizan el compromiso de distintos países en materia de cambio climático. 


\section{La continua adaptabilidad del principio de responsabilidades comunes pero diferenciadas}

Una segunda crítica al principio de responsabilidades comunes pero diferenciadas derivada de que la restrictiva interpretación del Protocolo de Kioto se basa en la inefectividad política (Bortscheller, 2010: 49; Brunnée y Streck, 2013: 594) y práctica (Andresen, 2014: 21-28; Bortscheller, 2010: 51; Brunnée y Streck, 2013: 594) del principio al lidiar con economías emergentes que se convirtieron en grandes emisores de $\mathrm{CO}_{2}$. Respecto a la inefectividad política, la ausencia de compromisos fijados para naciones con economías emergentes y emisiones en alza sirvió de argumento a Estados Unidos (Brunnée, y Streck, 2013: 594), el mayor país emisor entre 1992 y 2005, para no participar de dicho protocolo. En cuanto a la inefectividad práctica, el argumento radica en que la estática interpretación (Andresen, 2014: 27) que tuvo el principio de responsabilidades comunes pero diferenciadas generó efectos regresivos en la reducción de emisiones porque excluyó Estados con importantes niveles de emisión. Por ejemplo, China, México e India fueron parte de los diez países emisores más importantes ${ }^{10}$ sin embargo, ninguno de ellos tenía obligación alguna de reducir emisiones.

El contexto y los antecedentes anteriores promovieron la adopción de nuevas interpretaciones respecto del principio, fundamentalmente a través de nuevas posiciones adoptadas por los países en vías de desarrollo (Brunnée y Streck, 2013: 594). El Plan de Acción de Bali (ONU, 2008) orientó el régimen internacional del cambio climático hacia una visión más amplia de los compromisos climáticos (Rajamani, 2013). Sucesivamente, el Acuerdo de Copenhague (ONU, 2010) reconoció el principio, pero adoptó una interpretación matizada, llamando a los países que no están en el Anexo I a tomar medidas de mitigación (Brunnée, y Streck, 2013: 595). Sin embargo, el Acuerdo de Copenhague también estableció una clara y positiva distinción en favor de los países menos desarrollados y de los pequeños Estados insulares, instándolos a tomar compromisos voluntarios en la medida en que existiese apoyo y cooperación para ello. Fue en este contexto donde 43 países en vías de desarrollo fueron incorporados en los apéndices de esta decisión, entre ellos China como negociador clave (Hamdi-Cherif y Henri, 2016: 685). Estas naciones se comprometieron voluntariamente a tomar acciones de mitigación, ${ }^{11}$ constituyendo un paso importante en el régimen internacional del cambio climático. Similar visión matizada fue seguida por el Acuerdo de Cancún (ONU, 2011b), donde más países acordaron tomar acciones de

10. Global Carbon Atlas, disponible en http://www.globalcarbonatlas.org/en/CO2-emissions.

11. IEA, «The Twelfth Five-Year Plan for National Economic and Social Development of The Peoples Republic of China», 6 de julio de 2012, disponible https://bit.ly/2Zq9vNA; véase también Gupta, 2016: 171-174. 
mitigación. Finalmente, una interpretación distinta fue tomada en la Plataforma de Durban (ONU, 2011a), donde el principio no fue ni siquiera mencionado (Bodansky, Brunnée y Rajamani, 2017: 114).

Lo dicho ilustra cómo la adaptabilidad del principio responsabilidades comunes pero diferenciadas - que descansa en sus elementos comunes y diferenciados- jugó un rol esencial en al menos dos aspectos. En primer lugar, en el logro de acuerdos más efectivos y amplios dentro de las negociaciones del régimen internacional del cambio climático. La efectividad de dichos acuerdos descansa en el alto número de países en vías de desarrollo que asumieron obligaciones climáticas después del Acuerdo de Copenhague. Luego, el continuo reconocimiento del principio de responsabilidades comunes pero diferenciadas en distintas normas durante estos procesos de negociación (Rajamani, 2013: 154) garantizó el amplio apoyo que tuvieron estas decisiones.

En segundo lugar, el análisis también justifica que las críticas señaladas al principio de responsabilidades comunes pero diferenciadas solo se pueden circunscribir a su interpretación restrictiva, pero no al principio en sí. La limitada interpretación del principio en el Protocolo de Kioto implicó una fuente de críticas que dificultó la implementación de la equidad dentro del régimen internacional del cambio climático. Afortunadamente, la adaptabilidad del principio permitió superar dicha coyuntura.

$\mathrm{El}$ análisis anterior refleja nuevamente la esencialidad del principio de responsabilidades comunes pero diferenciadas, ya que una interpretación equivocada o una mala implementación pueden generar impactos negativos en el desarrollo del régimen climático. Al contrario, una interpretación y aplicación correcta y contextualizada de las responsabilidades comunes pero diferenciadas puede traer avances positivos en materia climática.

\section{El presente y futuro rol del principio de responsabilidades comunes pero diferenciadas}

En esta sección examinaremos la necesidad de conservar el principio de responsabilidades comunes pero diferenciadas para afrontar los futuros desafíos del cambio climático. Esto se desarrollará a partir de un análisis sobre la interpretación del principio en el Acuerdo de París y del impacto positivo que proyecta dicha interpretación en el régimen internacional del cambio climático.

\section{Contexto variable}

El contexto presente, y sobre el cual el régimen internacional del cambio climático se desarrolla, es significativamente distinto al de 1992 (Andresen, 2014: 28). El cre- 
cimiento de las economías de países en vías de desarrollo, ${ }^{12}$ el estado de emergencia del cambio climático (IPCC, 2018) y el incremento de la urbanización mundial ${ }^{13}$ han creado un contexto político nuevo donde han aparecido distintas alianzas (Brunnée y Streck, 2013: 599). En este escenario, la interpretación del principio de responsabilidades comunes pero diferenciadas juega un rol esencial en los acuerdos presentes y futuros. Por ejemplo, la interpretación matizada asumida por el régimen internacional del cambio climático después de Bali (Onu, 2008) ha sido entendida como una evolución en la efectividad del régimen (Andresen, 2014: 28), pero también como una erosión de la diferenciación (Rajamani, 2013: 169) porque ha tendido a igualar el rol de los países desarrollados y en vías de desarrollo. Estas posturas definen los debates presentes y futuros respecto del principio de responsabilidades comunes pero diferenciadas.

\section{El principio de responsabilidades comunes pero diferenciadas y el Acuerdo de París}

El Acuerdo de París tuvo el desafío de lograr un equilibrio entre un amplio apoyo de la comunidad internacional y el mantenimiento de una diferenciación equitativa (Voigt y Ferreira, 2016). Este balance fue reflejado en la Decisión de Lima, que llamó a las partes a un «acuerdo ambicioso», reconociendo el principio de responsabilidades comunes pero diferenciadas y considerando las «diferentes circunstancias nacionales» (ONU, 2015).

El Acuerdo de París aplicó el principio en cuatro campos principales: mitigación, adaptación, transparencia y financiamiento (Bodansky, Brunnée y Rajamani, 2017: 222-226). El principio es entendido en este acuerdo como un instrumento adaptable (Voigt y Ferreira, 2016: 66), donde los compromisos de las partes están en una situación de permanente evolución debido al constante control dentro del acuerdo (Rajamani, 2016: 503).

El Acuerdo de París es un buen ejemplo de cómo las instituciones pueden ser adaptadas teniendo como referencia su propia experiencia. De hecho, consideró todos los acuerdos y desacuerdos respecto al principio de responsabilidades comunes pero diferenciadas. Se convirtió, así, en un instrumento operativo y consensual y logró un «cuidadoso balance entre ambición y diferenciación» (Rajamani, 2016: 513).

En la línea de estos antecedentes, es innegable que el Acuerdo de París constituye una evidencia del entendimiento del principio como una herramienta que empuja a los países (Voigt y Ferreira, 2016: 74) a cumplir con los propósitos del régimen

12. World Bank, Data Bank, «World Development Indicators», disponible en https://bit.ly/3dHXSGT.

13. Organización de Naciones Unidas, World Urbanization Prospects: The 2018 Revision. Key Facts, disponible https://bit.ly/2BNXs4E. 
internacional del cambio climático. Esto confirma la necesidad de conservar y continuar mejorando la implementación del principio de responsabilidades comunes pero diferenciadas.

\section{El principio de responsabilidades comunes pero diferenciadas y su llegada a la COP25}

Hasta el momento hemos dado cuenta de la relevancia que el principio de responsabilidades comunes pero diferenciadas tiene para el régimen internacional del cambio climático a través del estudio de su evolución y adaptaciones a los distintos contextos. En este sentido, analizaremos el rol que este principio tuvo en la COP25.

Para hacer este análisis nos referiremos a la Conferencia Climática de Bonn ${ }^{14} \mathrm{de}$ junio 2019, preámbulo de la $\mathrm{COP}_{25}$, donde dos órganos del régimen internacional del cambio climático - el Cuerpo Subsidiario para la Implementación (CSI) ${ }^{15}$ y el Cuerpo Subsidiario de Consejo Científico y Tecnológico (CSCT) - ${ }^{16}$ discutieron los principales desafíos que la $\mathrm{COP}_{25}$ necesitaba definir. Entre estos temas estaba el peso de la evidencia científica en el desarrollo de las negociaciones climáticas (ONU, 2019b: 2) y la implementación del artículo 6 del Acuerdo de París que regula la cooperación internacional a través de instrumentos de mercado y de no-mercado. ${ }^{17}$ Así, esta sección tiene el propósito de analizar estos dos puntos durante la COP25, a la luz del rol que jugó el principio de responsabilidades comunes pero diferenciadas en su definición.

En primer lugar, abordaremos el peso de la evidencia científica en las negociaciones climáticas y su relación con el principio. En noviembre de 2018, el Panel Intergubernamental de Cambio Climático (IPCC por sus siglas en inglés) publicó un informe denominado Reporte Especial de 1,5 (IPCC, 2018), donde advirtió de la necesidad urgente de fijarse como meta principal 1,5 grados Celsius de tope en el aumento de la temperatura global. Sobre este objetivo, el artículo 2 (ONU, 2019b: 2) ${ }^{18}$ del Acuerdo de París establece una meta principal de 2 grados y una secundaria de 1,5 grados. Este reporte fue reconocido en la COP24 en su Decisión 1/CP.24 (ONU, 2019) como un instrumento que reforzaba el rol de la ciencia en el diseño de políticas para afrontar el cambio climático. Este reconocimiento planteado en Katowice también mandató al Cuerpo Subsidiario de Consejo Científico y Tecnológico para que considerará dicho informe en la Conferencia Climática de Bonn. En dicha conferencia, diversas naciones y organizaciones no gubernamentales insistieron con el lema «Science is

14. Naciones Unidas Cambio Climático, «Conferencia Climática de Bonn», disponible en https://bit. ly/2Zkfsf

15. CMNUCCC, artículo 10.

16. CMNUCC, artículo 9.

17. Acuerdo de París, artículo 6.

18. Acuerdo de París, artículo 2. 
not negotiable» (la ciencia no es negociable), sin embargo, la postura del Cuerpo Subsidiario de Consejo Científico y Tecnológico fue ambigua. Este órgano agradeció y reconoció el enorme aporte científico del Reporte, pero no determinó un peso o rol específico a la información revelada (ONU, 20919b: par. 84), postura que se mantuvo durante la $\mathrm{COP}_{25}$ (ONU, 2020).

Sobre lo presentado puede comentarse lo siguiente. Esta indefinición supone incertezas principalmente para los países más vulnerables a los efectos del cambio climático, estos son, los países menos desarrollados y los pequeños Estados insulares. El Panel Intergubenamental es claro al establecer que la alta vulnerabilidad frente al cambio climático está fuertemente influida por circunstancias no climáticas y desigualdades multidimensionales (IPCC, 2018: 6). De hecho, «personas que son social, económica, cultural, política, institucionalmente, o de otra manera, marginadas», son particularmente vulnerables a los efectos del cambio climático. Entonces, la protección de los países más vulnerables requiere la aplicación del principio de responsabilidades comunes pero diferenciadas. En este sentido, el primer paso para aplicar el principio en estos casos es reconocer y aplicar con claridad las certezas científicas que el Panel Intergubernamental ha aportado. Con seguridad, este punto seguirá siendo impulsado por muchos Estados parte de la Convención de cara a la COP26. La presidencia del Reino Unido deberá facilitar la definición de una postura que ponga a la ciencia como pilar esencial de futuras decisiones climáticas en materia de mitigación y adaptación; el principio de responsabilidades comunes pero diferenciadas debiera ser una guía útil para el logro de dicha definición.

Un segundo punto discutido en la Conferencia Climática de Bonn fue la implementación del artículo 6 del Acuerdo de París sobre cooperación entre las partes en materia de mitigación y adaptación. Existe una directa relación de los mecanismos de cooperación con el principio de responsabilidades comunes pero diferenciadas, ya que los primeros vienen a ser formas de implementación del segundo.

Para aclarar, son tres los mecanismos de cooperación establecidos en el artículo 6 del Acuerdo de París. Primero, el artículo 6.2 establece un mecanismo de cooperación bilateral y de mercado bajo el cual un país puede transferir voluntariamente a otro sus reducciones de emisiones. Segundo, el artículo 6.4 establece el Mecanismo de Desarrollo Sustentable, que viene a reemplazar el Mecanismo de Desarrollo Limpio y el de Implementación Conjunta que han operado durante la vigencia de Protocolo de Kioto. Tercero, el artículo 6.8 establece una cooperación basada en criterios de no-mercado. Dentro de las complicaciones que existen entre estos mecanismos se encuentra el riesgo de doble conteo (double-counting) de una misma reducción de emisiones. Por ejemplo, que al utilizarse el mecanismo del artículo 6.2 del Acuerdo de París, el crédito de carbono vendido por un país en desarrollo a un país desarrollado se considere en ambas contabilidades de reducción de emisiones. 
Lamentablemente, las negociaciones de la $\mathrm{COP}_{25}$ sobre este punto tuvieron un resultado negativo, y pospusieron esta resolución para la $\mathrm{COP}_{2} 6$ de Glasgow. Los desacuerdos se reflejaron principalmente en el debate sobre la consideración o no de créditos de carbono obtenidos bajo el Protocolo de Kioto (Masum, 2020: 11-12). Asimismo, la posibilidad de destinar la cooperación bilateral de mercado a financiamiento de adaptación, entre otros. ${ }^{19}$ En este sentido, se criticó que la presidencia chilena optara por sostener reuniones bilaterales con diferentes grupos para llegar a acuerdos, en vez de abordar con anterioridad dichos conflictos en plenario. Un buen punto de partida que la presidencia chilena pudo haber considerado era el inminente conflicto de diferenciación que la discusión del artículo 6 acarrearía. Un buen entendimiento del principio de responsabilidades comunes pero diferenciadas podría haber facilitado el diseño de una estrategia que pusiera este principio como elemento iluminador de los acuerdos.

Considerando lo anterior, resulta urgente lograr un acuerdo de implementación efectivo para disminuir emisiones. Es clave que el tipo de implementación que se defina respete el principio de equidad del régimen climático $y$, en consecuencia, el propio principio de responsabilidades comunes pero diferenciadas. El rol del principio dice relación con el establecimiento de un equilibrio entre la ambición que estos mecanismos de cooperación busquen y la efectiva protección y cooperación hacia de los países económica y climáticamente más vulnerables. Por ejemplo, la efectividad del mecanismo de control de doble conteo es clave para evitar, primero, un alza en el número global de emisiones, y, segundo, un incremento de los riesgos en países vulnerables al cambio climático que un doble conteo podría generar en el largo plazo. Finalmente, es necesario que la discusión sobre la implementación de este artículo no pierda su foco principal en Glasgow, esto es, en cuestiones de colaboración y desarrollo sustentable: esta es la clave del principio de responsabilidades comunes pero diferenciadas.

Los dos puntos comentados son relevantes ya que influyen directamente en la implementación del principio. Un ejemplo que ilustra su importancia es el escenario de riesgos de los pequeños Estados insulares. Estos países son vulnerables a inundaciones por incremento del nivel del mar, por tanto, necesitan fuertes compromisos de mitigación de los países con más emisiones y una notable cooperación de los Estados desarrollados destinada a la ejecución de proyectos de adaptación. En este sentido, tanto el valor de las proyecciones científicas hechas por el Panel Intergubernamental como la definición de la implementación del artículo 6 del Acuerdo de París, resultan urgentes de resolver para contrarrestar los efectos del cambio climático y entregar mayores certezas a estas naciones insulares.

19. Véase European Capacity Building Initiative, COP 25. Key outcomes, disponible en https://bit. ly/3gdxZAm, p. 8. 
En conclusión, la $\mathrm{COP}_{25}$ era una instancia decisiva para alcanzar acuerdos en los puntos mencionados. Claramente constituyó una oportunidad perdida respecto a las materias recién mencionadas. En este sentido, hay mucha esperanza puesta en la $\mathrm{COP}_{26}$, donde se requiere que predomine una visión de equidad y protectora de los países más vulnerables al cambio climático. Cumplir con este requerimiento implica una aplicación del principio de responsabilidades comunes pero diferenciadas, acorde a la visión ambiciosa y diferenciadora que el Acuerdo de París representa.

\section{Conclusiones}

En conclusión, el principio de responsabilidades comunes pero diferenciadas es un elemento esencial en los orígenes, evolución y proyección del régimen internacional del cambio climático. Además, desde una perspectiva de equidad, es una herramienta clave para reducir los efectos que el cambio climático provocará en países más vulnerables.

La arquitectura del Acuerdo de París definió un camino donde el principio es esencial para el desarrollo del régimen climático, entendiendo este principio como un «catalizador [...] de la acción climática» (Voigt y Ferreira, 2016: 74). Por tanto, con el Acuerdo de París, el principio de responsabilidades comunes pero diferenciadas recupera sus elementos dinámicos naturales (Rajamani, 2012: 134).

El desafío está principalmente relacionado con el cuidado de evitar interpretaciones desequilibradas del principio de responsabilidades comunes pero diferenciadas. Ciertamente, es evidente que interpretaciones restrictivas (Bodansky, Brunnée y Rajamani, 2017: 27) basadas en categorías imperativas ${ }^{20}$ pueden disminuir el consenso y debilitar el régimen internacional del cambio climático. De esta manera, una interpretación matizada de este principio es fundamental para continuar mejorando el compromiso de las Partes y la efectividad de sus acciones. Al mismo tiempo, la estabilidad del régimen descansa en un reconocimiento equitativo de las diferencias (Winkler y Rajamani, 2014: 102-103), así como en una extrema flexibilidad en la interpretación de este principio puede erosionar su propósito y el régimen internacional de cambio climático. En este sentido, el control continuo de las diferentes circunstancias nacionales ${ }^{21}$ es esencial para proteger el requerido balance que el principio de responsabilidades comunes pero diferenciadas necesita para ser justo, efectivo y operativo dentro del régimen internacional del cambio climático.

20. Ver UNFCCC, arts. 4.1, 4.2, 4.3, 4.5, 5, 6, 12.1, 12.2 citados en Bodansky, Brunnée y Rajamani (2017: 122).

21. Ver, Acuerdo de París, art.14 


\section{Referencias}

Andresen, Steinar (2014). «The Climate Regime: A Few Achievements, but Many Challenges». Climate Law 4 (1-2): 21-28. Disponible en https://bit.ly/2YKlCWU.

AGNU, Asamblea General de Naciones Unidas (1989a). Conferencia de las Naciones Unidas sobre Medio Ambiente y Desarrollo.

-. (1989b). Protección del clima mundial para las generaciones presentes y futuras, $\mathrm{p}$. 9. Acceso el 19 de noviembre de 2018.

-. (1991a). Report of the Intergovernmental Negotiating Committee for a Framework Convention on Climate Change on the work of its First Session.

-. (1991b). Report of the Intergovernmental Negotiating Committee for a Framework Convention on Climate Change on the work of its Third Session.

-. (1991c). Report of the Intergovernmental Negotiating Committee for a Framework Convention on Climate Change on the work of its Fourth Session.

-. (1992). Informe de la Conferencia de las Naciones Unidas sobre el Medio Ambiente y el Desarrollo. Vol. I.

Atapattu, Sumudu. 2009. "Climate change, differentiated responsibilities and state responsibility: devising novel legal strategies for damage caused by climate change», p. 37. En Climate Law and Developing Countries: Legal and Policy Challenges for the World Economy, editado por Benjamin J Richardson, Yves Le Bouthilier, Heather McLeod-Kilmurray, \& Stepan Wood. Edward Elgar Publishing.

Birnie, Patricia, Alan Boyle y Catherine Redgwell (2009). International Law and the Environment. Tercera edición. Londres: Oxford University Press.

Bodansky, Daniel, Jutta Brunnée y Lavanya Rajamani (2017). International Climate Change Law. Londres: Oxford University Press.

Bortscheller, Mary J. (2010). «Equitable but Ineffective: How the Principle of Common but Differentiated Responsibilities Hobbles the Global Fight against Climate Change». Sustainable Development Law \& Policy 10 (2): 49-69. Disponible en https://bit.ly/3eIXZUc.

Brown Weiss, Edith (2007). «Climate Change, Intergenerational Equity, and International Law». Vermont Journal of Environmental Law, 9: 615-628. Disponible en https://bit.ly/3icqOdH.

BRUnNÉE, Jutta y Charlotte Streck (2013). «The UNFCCC as a Negotiation Forum: Towards Common but More Differentiated Responsibilities». Climate Policy, 13 (5): 589-607. Disponible en https://bit.ly/2NImSUa.

CARroll, John E. (1988). International Environmental Diplomacy: The Management and Resolution of Transfrontier Environmental Problems. Cambridge University Press.

DinAH, Shelton (2008). «Equity». En The Oxford Handbook of International Environmental Law, editado por Daniel Bodansky, Jutta Brunnée, and Ellen Hey. Oxford University Press. 
French, Duncan (2000). «Developing States and International Environmental Law: The Importance of Differentiated Responsibilities». International and Comparative Law Quarterly 49 (1): 35-60.

Gupta, Joyeeta (2016). «The Paris Climate Change Agreement: China and India». Climate Law 6 (1-2): 171-181.

Hamdi-Cherif, Meriem y Henri Waisman (2016). «Global Carbon Pricing and the 'Common but Differentiated Responsibilities': The Case of China». International Environmental Agreements: Politics, Law and Economics 16, $\mathrm{N}^{\circ}{ }_{5}$ : 671-689. https:// link.springer.com/article/10.1007/s10784-015-9289-2

IPCC, Intergovernmental Panel on Climate Change (2014). «Summary for policymakers». En Climate Change 2014: Impacts, Adaptation, and Vulnerability. Contribution of Working Group II to the Fifth Assessment Report of the Intergovernmental Panel on Climate Change. Cambridge University Press, Cambridge, United Kingdom y New York, Estados Unidos. https://www.ipcc.ch/site/assets/ uploads/2018/02/ar5_wgII_spm_en.pdf

-. (2018). Global Warming of $1,5{ }^{\circ} \mathrm{C}$. An IPCC Special Report on the impacts of global warming of $1.5^{\circ} \mathrm{C}$ above pre-industrial levels and related global greenhouse gas emission pathways, in the context of strengthening the global response to the threat of climate change, sustainable development, and efforts to eradicate poverty. Ginebra: Organización Mundial de Metereología. https://www.ipcc.ch/site/assets/uploads/ sites/2/2019/05/SR15_SPM_version_report_LR.pdf.

Jamieson, Dale (2003). «Ethics, Public Policy and Global Warming». En Morality's Progress. Oxford University Press.

Gardiner, Stephen M. (2004). «Ethics and global climate change». Ethics 114 (3): 555-600.

Masum, Jahangir Hasan (2020). An Overview of the 25th Conference of the Parties (COP) to the United Nations Framework Convention on Climate Change (UNFCCC). Coastal Development Partnership (CDP).

Mickelson, Karin (2008). «Critical Approaches». En Daniel Bodansky, Jutta Brunnée, y Ellen Hey (editores), The Oxford Handbook of International Environmental Law. Oxford University Press.

ONU, Organización de las Naciones Unidas (1972). Informe de la Conferencia de las Naciones Unidas sobre el Medio Humano. «Declaración de Estocolmo» https:// www.dipublico.org/conferencias/mediohumano/A-CONF.48-14-REV.1.pdf

-. (2008). Informe de la Conferencia de las Partes sobre su $13^{\circ}$ período de sesiones, celebrado en Bali del 3 al 15 de diciembre de 2007. Decisión 1/CP.13, 3. https://unfccc. int/resource/docs/2007/cop13/spa/o6ao1s.pdf

-. (2010). Informe de la Conferencia de las Partes sobre su $15^{\circ}$ período de sesiones, celebrado en Copenhague del 7 al 19 de diciembre de 2009. Decisión 2/CP.15, «Acuerdo de Copenhague». https://unfccc.int/sites/default/files/resource/docs/2009/cop15/ 


\section{spa/11ao1s.pdf?download}

-. (2011a). Establecimiento de un Grupo de Trabajo Especial sobre la Plataforma de Durban para una acción reforzada. Decisión 1/CP.17. https://unfccc.int/sites/default/files/resource/docs/2011/cop17/spa/lios.pdf?download

-. (2011b). Informe de la Conferencia de las Partes sobre su $16^{\circ}$ período de sesiones, celebrado en Cancún del 29 de noviembre al 10 de diciembre de 2010. Decisión 1/ CP.16. https://unfccc.int/resource/docs/2010/cop16/spa/o7ao1s.pdf

-. (2015). Informe de la Conferencia de las Partes sobre su $20^{\circ}$ período de sesiones, celebrado en Lima del 1 al 14 de diciembre de 2014. Decisión 1/CP2o. https://unfccc. int/sites/default/files/resource/docs/2014/cop2o/spa/10ao1s.pdf

-. (2016). Informe de la Conferencia de las Partes sobre su $21^{\circ}$ período de sesiones, celebrado en París del 30 de noviembre al 13 de diciembre de 2015. Decisión 1/CP.21, «Adopción del Acuerdo de París». https:/unfccc.int/sites/default/files/resource/ docs/2015/cop21/spa/10ao1s.pdf? download

-. (2019a). Informe de la Conferencia de las Partes sobre su $24^{\circ}$ período de sesiones, celebrado en Katowice del 2 al 15 de diciembre de 2018. Disponible en https://undocs. org/es/FCCC/CP/2018/10/ADD.1.

-. (2019b). Informe del Órgano Subsidiario de Asesoramiento Científico y Tecnológico sobre su $50^{\circ}$ período de sesiones, celebrado en Bonn del 17 al 27 de mayo de 2019. Disponible en https://unfccc.int/sites/default/files/resource/sbsta2019_02S.pdf.

-. (2020). Informe de la Conferencia de las Partes en calidad de reunión de las Partes en el Acuerdo de París sobre su segundo período de sesiones, celebrado en Madrid del2al15 de diciembre de 2019. Decisión 1/CMA.2. Disponible https://bit. ly/zeRhBWk.

Rajamani, Lavanya (2000). «The Principle of Common but Differentiated Responsibility and the Balance of Commitments under the Climate Regime». Review of European Community \& International Environmental Law 9: 120-131.

-. (2012). Differential Treatment in International Environmental Law. Oxford University Press.

-. (2013). «Differentiation in the Emerging Climate Regime Reaching International Cooperation on Climate Change Mitigation». Theoretical Inquiries in Law, 14 (19): 151-172.

-. (2016). «Ambition and differentiation in the 2015 Paris Agreement: Interpretative possibilities and underlying politics». International and Comparative Law Quarterly 65 (2): 493-514.

Rowlands, Ian H. (2008). "Atmosphere and Outer Space». En Daniel Bodansky, Jutta Brunnée y Ellen Hey (editores), The Oxford Handbook of International Environmental Law. Oxford University Press.

SAnds, Philippe (2003). Principles of International Environmental Law. Cambridge University Press. 
SHUE, Henry (1999). «Global Environment and International Inequality». International Affairs 75 (3): 531-545. Disponible en https://bit.ly/2Vx5RjX.

-. (2016). Climate Justice: Vulnerability and Protection. Oxford University Press.

Soltau, Frederiech (2016). «Common Concern of Humankind». En Kevin R Gray, Richard Tarasofsky y Cinnamon Carlarne (editores), The Oxford Handbook of International Climate Change Law. Oxford University Press.

Stern, Nicholas (2010). «The Economics of Climate Change». En P. Singer, S. M. Gardiner, S. Caney, D. Jamieson y H. Shue (editores), Climate Ethics: Essential Readings, Oxford University Press.

STEWART, Richard B. (1992). «Environmental Regulation and International Competitiveness Articles and Comment». Yale Law Journal, 102: 2039-2106. Disponible en https://bit.ly/2BnURil.

van der GaAst, Wytze (2017). «The First Phase. Negotiating the UN Climate Convention». En Wytze van der Gaast (editor), International Climate Negotiation Factors (pp. 43-55). Springer International Publishing.

VoIgt, Christina y Felipe Ferreira (2016). «Differentiation in the Paris Agreement». Climate Law 6 (1-2): 58-74.

Weisslitz, Michael (2002). «Rethinking the Equitable Principle of Common but Differentiated Responsibility: Differential versus Absolute Norms of Compliance and Contribution in the Global Climate Change Context Notes and Comments». Colorado Journal of International Environmental Law and Policy, 13: 473-510.

Werksman, Jacob. (1998). "The Clean Development Mechanism: Unwrapping the 'Kyoto Surprise'». Review of European Community \& International Environmental Law, 7: 147-158.

Weston, Burns H. (2007). «Climate Change and Intergenerational Justice: Foundational Reflections» Vermont Journal of Environmental Law, 9: 375-430.

WINKLER, Harald y Lavanya Rajamani (2014). «CBDR\&RC in a Regime Applicable to All». Climate Policy 14 (1): 102-121. Disponible en https://bit.ly/2YI6byg.

Yamin, Farhana. 1998. «The Kyoto Protocol: Origins, Assessment and Future Challenges». Review of European Community \& International Environmental Law 7: 113-122.

\section{Sobre el autor}

Pedro Cisterna Gaete es abogado de la Universidad de Concepción. LlM Global Environment and Climate Change Law, Universidad de Edimburgo. Estudiante de doctorado en Derecho, Universidad de Edimburgo. Su correo electrónico es pedro.cisternag@gmail.com. (D) https://orcid.org/0000-0002-5527-9892. 
La Revista de Derecho Ambiental, del Centro de Derecho Ambiental de la Facultad de Derecho de la Universidad de Chile, es un espacio de exposición y análisis en el plano académico del derecho ambiental. Su contenido se presenta a través de doctrina, jurisprudencia y recensiones, y aborda diversas materias relacionadas con la gestión, institucionalidad y herramientas de protección ambiental y desarrollo sustentable. Se presentan artículos de diferentes autores, en los que se analizan y abordan casos y temas jurídico-ambientales de creciente interés y actualidad.

\author{
DIRECTORA \\ Valentina Durán Medina \\ EDITORES \\ Jorge Ossandón Rosales \\ y Antonio Pulgar Martínez \\ SITIO WEB \\ revistaderechoambiental.uchile.cl \\ CORREO ELECTRÓNICO \\ revistada@derecho.uchile.cl \\ LICENCIA DE ESTE ARTÍ́CULO
}

Creative Commons Atribución Compartir Igual 4.o Internacional

La edición de textos, el diseño editorial

y la conversión a formatos electrónicos de este artículo

estuvieron a cargo de Tipográfica

(www.tipografica.io). 